\title{
L'émergence du modèle coopératif en Corée du Sud
} The emergence of the cooperative model in South Korea La emergencia del modelo cooperativo en Corea del Sur

\section{Eric Bidet et Hyungsik Eum}

Numéro 341, juillet 2016

\author{
Asie \\ Asia \\ URI : https://id.erudit.org/iderudit/1037435ar \\ DOI : https://doi.org/10.7202/1037435ar
}

Aller au sommaire du numéro

Éditeur(s)

Association Recma

ISSN

1626-1682 (imprimé)

2261-2599 (numérique)

Découvrir la revue

\section{Citer cet article}

Bidet, E. \& Eum, H. (2016). L'émergence du modèle coopératif en Corée du Sud. Revue internationale de l'économie sociale, (341), 26-43.

https://doi.org/10.7202/1037435ar

\section{Résumé de l'article}

Depuis une quinzaine d'années, et spécialement depuis l'introduction d'une loi coopérative générale en 2012, on assiste en Corée à un renouveau très intéressant du modèle coopératif. Celui-ci, qui a longtemps été identifié à une sorte d'agence publique en référence aux coopératives traditionnelles du secteur primaire et bancaire mises en place par l'Etat dans les années 1960 tend désormais à être reconnu comme un modèle d'entreprise à part entière possédant certaines vertus, notamment en matière de création d'emplois ou de développement territorial. Cet article analyse l'émergence de cet intérêt nouveau pour le modèle coopératif en montrant comment il s'est construit de façon progressive à partir d'expériences pionnières apparues à partir de la fin des années 1980, de politiques publiques visant à promouvoir l'entreprise sociale, jusqu'à l'adoption de la loi de 2012 qui a généré un " choc coopératif ». 


\title{
L'ÉMERGENCE DU MODĖLE COOPÉRATIF EN CORÉE DU SUD
}

\author{
par Eric Bidet* et Hyungsik Eum**
}

\begin{abstract}
Depuis une quinzaine d'années, et spécialement depuis l'introduction d'une loi coopérative générale en 2012, on assiste en Corée à un renouveau très intéressant du modèle coopératif. Celui-ci, qui a longtemps été identifié à une sorte d'agence publique en référence aux coopératives traditionnelles du secteur primaire et bancaire mises en place par l'Etat dans les années 1960, tend désormais à être reconnu comme un modèle d'entreprise à part entière possédant certaines vertus, notamment en matière de création d'emplois ou de développement territorial. Cet article analyse l'émergence de cet intérêt nouveau pour le modèle coopératif en montrant comment il s'est construit de façon progressive à partir d'expériences pionnières apparues à partir de la fin des années 1980, de politiques publiques visant à promouvoir l'entreprise sociale, jusqu'à l'adoption de la loi de 2012 qui a généré un «choc coopératif».
\end{abstract}

\section{The emergence of the cooperative model in South Korea}

For the past fifteen years, and especially since the law on cooperatives in 2012, there has been a very interesting revival of the cooperative model in Korea. For a long time seen as a sort of public agency like the traditional cooperatives set up by the government in the primary and banking sectors in the 1960s, cooperatives are now recognised as an independent firm model with certain advantages, particularly in terms of job creation and local development. This article analyses the new interest in the cooperative model by showing how it gradually developed starting from the pioneering experiences in the late 1980s of public policies aimed at promoting social enterprise through to the 2012 law which produced a cooperative boom.

\section{La emergencia del modelo cooperativo en Corea del Sur}

Desde hace unos quince años, y especialmente desde la introducción en 2012 de una ley cooperativa general, se observa un renacimiento muy interesante del modelo cooperativo en Corea. Este fue identificado desde hace mucho tiempo a un tipo de agencia pública por referencia a las cooperativas tradicionales del sector primario y bancario establecidas por el Estado en los años 60s. Ahora tiende a ser reconocido como un modelo de empresa de pleno derecho teniendo ciertas virtudes, particularmente en materia de creación de empleos o de desarrollo territorial. El presente artículo analiza la emergencia de este nuevo interés para el modelo cooperativo y muestra cómo fue construido de manera progresiva a partir de experiencias pioneras aparecidas al final de los años 80s, de políticas públicas dirigidas a promover la empresa social, hasta la aprobación de la ley de 2012, que ha generado un "choque cooperativo".

\footnotetext{
* Responsable du master Economie sociale et solidaire, université du Mans.

** Doctorant en sociologie, université de Liège.
} 

sociale ont été identifiées et fortement encouragées par les pouvoirs publics pour la mise en œuvre d'activités à finalité sociale ou en lien avec des problématiques sociales. Le gouvernement sud-coréen s'est notamment doté en 2006 d'une loi pour la promotion de l'entreprise sociale pour en faire un des leviers de sa politique d'emploi et de lutte contre la pauvreté et l'exclusion, puis de sa politique en direction des communautés locales. Au total, c'est un ensemble assez complexe de dispositifs qui ont été installés en une quinzaine d'années, reflétant une dynamique peu commune impliquant étroitement les pouvoirs publics, tant au niveau national que local (Eum, 2008; Eum, Bidet, 2014). Différents ministères ont ainsi mis en place des politiques visant à encourager le modèle de l'entreprise sociale ou de l'économie sociale à travers un vaste réseau d'agences locales chargées de la mise en œuvre de ces politiques. Les différentes collectivités territoriales (provinces, municipalités) ont adopté des décrets allant également dans ce sens et dégagé des moyens spécifiques pour encourager les réponses à des problématiques locales liées à l'emploi, à l'agriculture, aux services à la personne, à la restauration scolaire, au logement, etc. La finalité sociale de ces expériences justifie pleinement, en général, qu’elles reçoivent un soutien public, qui est d'ailleurs souvent nécessaire à leur émergence et à leur pérennisation.

Jusqu’à récemment, le modèle coopératif était relativement peu mis en avant dans ces démarches, qui restaient fortement liées à la poursuite d'une finalité sociale et cherchaient le plus souvent à articuler ressources publiques et ressources privées pour amener la sortie du chômage, par l'appui à la création d'activité, par l'acquisition de qualifications ou par l'accompagnement des travailleurs peu qualifiés. Les évolutions récentes montrent un intérêt nouveau pour le modèle coopératif, à la fois dans la continuité de celui pour les organisations à finalité sociale (coopératives sociales), mais également comme modèle d'entreprise à gouvernance participative (jusqu'à l'adoption de règles de distribution égalitaire des droits de vote entre les membres), susceptible de se développer dans pratiquement toutes les activités économiques (coopératives de consommateurs et coopératives dites " génériques »). Cet article analyse l'émergence de cet intérêt nouveau pour le modèle coopératif en montrant comment il s'est construit de façon progressive à partir de différentes expériences spécifiques apparues successivement depuis la fin des années 1980, jusqu'à l'adoption d'une loi coopérative générale en 2012. Pour réaliser cette étude, nous nous sommes principalement appuyés sur l'analyse des textes législatifs en vigueur, sur les travaux publiés 
en Corée sur la question et sur des entretiens et visites de terrain réalisés dans des coopératives et fédérations de coopératives dans plusieurs villes coréennes.

\section{Le développement des coopératives de consommation}

Comme cela été été analysé dans de précédents articles (Bidet, 2008; Eum, Bidet, 2014), il se dégage un consensus pour considérer que les principales réalisations coopératives significatives et pérennes en Corée datent du début des années 1960. C’est en effet à cette époque que sont mis en place, à l'initiative du gouvernement, les premiers cadres législatifs qui vont servir à structurer les mouvements coopératifs du secteur primaire (agriculture, élevage, pêche, forêts) et leur déclinaison dans le secteur secondaire (industrie agroalimentaire) et le secteur tertiaire (banque). Au cours des décennies suivantes, ces mouvements ont connu plusieurs restructurations, qui ont amené des regroupements et fait de la Fédération nationale des coopératives agricoles (NACF) un puissant acteur du monde coopératif à l'échelle internationale (avec environ 2,5 millions de membres et quelque 30000 employés, la NACF fait partie du top 10 des coopératives au niveau mondial) et, bien évidemment, à l'échelle nationale. Etroitement contrôlés par les pouvoirs publics jusqu’à la fin des années 1980, ces mouvements ont contribué à associer à la coopérative l'image d'une organisation quasi gouvernementale, sorte d'agence publique servant des stratégies électorales et utilisée pour la mise en œuvre de certaines politiques publiques (notamment dans le domaine agricole). Ces coopératives traditionnelles ont entrepris de s'affranchir de leur tutelle publique à la faveur de la démocratisation politique du pays, à partir de la fin des années 1980. Elles continuent néanmoins d’incarner un modèle coopératif très bureaucratique, inspiré à la fois par le modèle de l'entreprise publique et par celui des banques traditionnelles, avec lesquelles elles sont en concurrence, car elles tirent l'essentiel de leurs ressources de leur activité bancaire et financière.

La première tendance qui incarne une vision différente du modèle coopératif issu de la base et reposant sur une gestion participative est celle des coopératives de consommation. Après plusieurs tentatives, souvent éphémères, qui apparaissent à partir des années 1920, la première expérience significative est celle de la coopérative Shinri, qui naît à la fin des années 1970 à l'initiative de dirigeants issus du mouvement des credit unions. Elle sera suivie de quelques autres initiatives qui génèrent, au milieu des années 1980, un premier regroupement informel, duquel sortira en 1987 la première Fédération nationale des coopératives de consommation, organisme à but non lucratif 
reconnu par le ministère de l'Economie. Cette fédération contribuera à une meilleure reconnaissance des coopératives de consommation, jusqu'à l'adoption d'une loi spécifique en 1999. Des initiatives variées sont expérimentées à partir du milieu des années 1980. A la différence de tentatives plus anciennes, mais qui demeurent assez éphémères, ces initiatives mettent l'accent sur l'environnement, la solidarité avec les petits agriculteurs, le développement communautaire, le droit des consommateurs, etc. C'est la raison pour laquelle ces coopératives vont essentiellement baser leur activité sur la promotion de produits issus de l'agriculture biologique dans une démarche comparable à celle que mène Biocoop en France. Le terme choisi en coréen pour désigner ces coopératives de consommation est celui de saenghyeop, dont une traduction plus ou moins exacte serait «coopératives pour la vie quotidienne». Cela exprime le fait que ces coopératives se sont développées sous des formes plus variées que celles que l'on connaît en général en Europe, incluant par exemple des coopératives universitaires (dont les membres sont des étudiants, des enseignants et des employés d'universités) ou des coopératives médicales, sur lesquelles nous reviendrons.

Les idées qui sont développées à Wonju à partir des années 1960 jouent un rôle essentiel dans cette orientation de la coopération de consommation coréenne. Ville de 270000 habitants située dans la province du Gangwon, à environ 100 kilomètres à l'est de Séoul, Wonju est l'un des foyers coopératifs les plus dynamiques en Corée et, durant la période de dictature (1955-1987), l'un des principaux centres de contestation sociale sous l'influence de deux des grandes figures du mouvement social coréen, le prêtre Ji Hak-sun et l'éducateur Jang Il-soon. Tous deux sont catholiques et illustrent le rôle central joué en Corée par l'Eglise catholique dans la lutte pour la démocratie. Dans les années 1960, la région de Wonju est l'un des centres de l'industrie minière, tout en restant une région très rurale comme l'est encore la majeure partie du pays à l'époque (le secteur primaire rassemble plus de $50 \%$ de la population et représente encore $40 \%$ du PIB en 1965). Sont expérimentées à Wonju différentes initiatives pour tenter de répondre aux problèmes rencontrés par les paysans accablés par l'usure et les catastrophes climatiques (notamment les inondations autour de la rivière Namhan en 1972).

A partir de Wonju ont essaimé dans la région du Gangwon les idées de la coopération de crédit en milieu rural, de la coopération de production, de la coopération pour l'utilisation en commun du matériel agricole. Apparaissent également des démarches précurseurs de la coopération de consommation, y compris celle de Shinri, pour commercialiser des produits nécessaires aux ouvriers des mines (vêtements, gants, bottes, etc.) et pour faire valoir leurs droits. On retrouve dans les initiatives apparues 
à Wonju certains traits des expériences coopératives pionnières en Europe au XIX siècle, illustrant le fait qu'il s'agit d'un mouvement social portant un projet politique tourné vers les besoins des classes populaires et inspiré par la recherche d'une meilleure justice sociale. Les besoins auxquels essaient de répondre ces initiatives et les réponses qu'elles proposent sont globalement les mêmes que ceux observés en Europe un siècle et demi plus tôt. A partir de la fin des années 1970, Wonju joue également un rôle pionnier dans la dénonciation de l'utilisation massive d'engrais chimiques, dont les coopératives agricoles traditionnelles sont un des principaux vecteurs, et des problèmes sanitaires (pollution, maladies) et sociaux (exode rural) qui en découlent. Sont ainsi posées à Wonju les bases qui vont servir en Corée au développement de la coopération de consommation et du mouvement écologiste, deux directions qu'incarne le mouvement Hansalim.

Dans les années 1980, Jang Il-soon défend à Wonju une forme d'agriculture alternative basée sur le principe d'une gouvernance participative associant petits producteurs ruraux et consommateurs urbains, au service du développement communautaire. L'idée fondamentale, développée dans le Manifeste Hansalim de 1989, est celle d'une responsabilité partagée entre producteur et consommateur: le premier est responsable de la santé du second, tandis que le second est responsable de la survie du premier. Hansalim se développe ensuite à partir de Séoul et des grandes métropoles dans une démarche qui repose sur l'idée de construire un lien social entre des consommateurs des grands centres urbains, concernés par la santé et l'environnement, et des petits producteurs qui souhaitent sortir d'un système d'agriculture intensive reposant sur l'utilisation massive d'engrais chimiques. Cette idée inspire ensuite d'autres démarches, ce qui donne naissance à un mouvement particulièrement dynamique de coopératives de consommation étroitement liées à l'agriculture biologique et, plus largement, aux questions d'environnement et de santé. La démarche présente des similitudes avec celles développées en France par le réseau Biocoop ou par les associations pour le maintien d'une agriculture paysanne (Amap), elles-mêmes inspirées par le modèle japonais des Teikei. Hansalim apparaît cependant plus proche d'un autre mouvement japonais: celui des coopératives de consommation japonaises, notamment du Seikatsu Club, avec lequel il partage notamment une caractéristique essentielle qui est de reposer sur un sociétariat et un engagement bénévole fortement féminisés (même si ses leaders, actuels et passés, sont surtout des hommes).

A la fin des années 1990, alors que le pays traverse une grave crise financière, les coopératives de consommateurs se regroupent dans des réseaux nationaux dont les plus importants sont aujourd'hui Hansalim, Icoop et Dure, qui comptent à eux 
trois plus de 100000 adhérents (ICA Committee on Consumer Cooperation for Asia and Pacific, 2012). En 2007, on estimait que les différents réseaux de coopératives de consommation totalisaient quelque 400000 membres, dont plus du quart (115000) au sein du réseau Hansalim. Tous ont pour caractéristique commune d'avoir construit leur activité autour de la commercialisation de produits issus de l'agriculture biologique, et donc de promouvoir la coopération entre des consommateurs et des agriculteurs. Ils jouent de ce fait un rôle pionnier dans le domaine de l'agriculture biologique, en introduisant notamment des normes spécifiques, et dans la promotion des circuits courts (localfood). Aujourd'hui, on estime qu'ils représentent environ $30 \%$ du marché bio en Corée. En 2010, ces différents mouvements avaient un sociétariat cumulé d'environ 700000 membres et un réseau de distribution de plus de 300 points de vente, sachant que leur principal canal de distribution est la vente sur Internet avec livraison à domicile. On remarquera que ces chiffres sont très comparables à ceux indiqués pour la France par CoopFR. Sur la décennie 2000-2010, les coopératives coréennes de consommateurs ont enregistré un taux de croissance annuel moyen d'environ $35 \%$. Hansalim, par exemple, a vu son chiffre d'affaires passer de l'indice 100 en 2000 à l'indice 1000 en 2010. En 2015, Icoop comptait environ 220000 membres, Hansalim près de 350000 (sociétariat comparable à celui de Coop Atlantique, la plus grande coopérative de consommation en France).

La reconnaissance légale des coopératives de consommation à la fin des années 1990 et le succès important qu'elles rencontrent durant la décennie suivante, répondant notamment à l'engouement massif pour les produits naturels, valorisés dans les stratégies marketing des principaux opérateurs commerciaux à travers le concept de well-being, ont contribué à modifier l'image des coopératives pour en faire progressivement celui d'un modèle d'entreprise spécifique, autonome et capable d'apporter une réponse pertinente à certaines problématiques en lien avec l'agriculture biologique, l'alimentation saine, la préservation, ou encore la transformation de petites exploitations agricoles, la restauration scolaire et les circuits courts. L'expérience très spécifique des coopératives médicales (ou coopératives de santé) a joué également un rôle particulier en tant que forme particulière de coopérative de consommation reconnue par la loi de 2006 pour la promotion de l'entreprise sociale.

\section{L'expérience emblématique des coopératives médicales}

Avec cette loi adoptée fin 2006, la Corée devient l'un des tout premiers pays à se doter d'un cadre législatif propre aux entreprises sociales, incluant notamment une définition 
de l'entreprise sociale conçue non pas comme un statut juridique spécifique, mais comme un modèle d'entreprise susceptible d'être mis en œuvre à travers différents statuts juridiques à but lucratif ou non lucratif. Cette loi est un dispositif de labélisation contrôlé par le gouvernement, plus particulièrement par le ministère du Travail, qui attribue la reconnaissance en tant qu'entreprise sociale et contrôle ainsi de manière exclusive l'usage de l'appellation « entreprise sociale » (une organisation non reconnue par la loi de 2006 n'est pas autorisée à utiliser cette appellation). La finalité de l'organisation est le point d'entrée principal de cette reconnaissance: pour être reconnue comme entreprise sociale, une structure doit avoir comme finalité principale l'emploi de personnes en difficulté, la fourniture de services à des catégories de personnes en difficulté ou la réponse à des besoins communautaires. Ces conditions sont assez proches de celles qui définissent en France l'utilité sociale dans la loi de 2014 sur l'ESS. Début 2016, l'agrément « entreprise sociale » avait été accordé en Corée à un peu plus de 1500 structures, soit une moyenne annuelle de plus de 150 habilitations depuis l'entrée en vigueur de la loi, début 2007. Les coopératives médicales sont l'une des formes d'organisations qui ont bénéficié de cet agrément.

La première coopérative médicale coréenne a été créée en 1994 dans la ville d’Anseong (80 kilomètres au sud de Séoul, 190000 habitants) à l'initiative d'un groupe de paysans et d'une association d'étudiants en médecine désireux de s'engager bénévolement pour dispenser des soins médicaux à une population incapable d'en assumer le coût dans le système traditionnel. Faute de disposer d'un cadre juridique spécifique, elle adopte le statut associatif. Cette première initiative est inspirée du modèle japonais, où les coopératives de santé sont apparues dans les années 1940 et se sont organisées en fédération dès les années 1950 (elles ont ensuite connu un remarquable développement, puisqu'on en compte aujourd'hui plus de cent, qui emploient plus de 30000 personnes). La suivante est créée deux ans plus tard à Incheon (grande banlieue de Séoul, 2,7 millions d'habitants) par une association de jeunes médecins protestants, suivie d'une troisième en 2000, à Ansan (agglomération de Séoul, 750000 habitants), par des mouvements de citoyens en collaboration avec des membres d'un centre de recherche en médecine. L'expérience de la coopérative médicale de Daejeon, créée en 2002, est particulièrement intéressante, car elle se développe à l'initiative d'un groupe de citoyens qui ont développé un système de monnaie complémentaire locale (Hanbat Lets). Le réseau des adhérents à Hanbat Lets va constituer pendant les premières années l'essentiel des adhérents à la coopérative médicale, jusqu'à ce que celle-ci touche une population plus large en faisant le choix de s'implanter dans 
des quartiers plus populaires. Inspirée par l'expérience et les valeurs de Hanbat Lets, la coopérative développe une approche de la médecine basée sur la participation responsable. Les différents associés participent à la gouvernance de la clinique, qui propose des soins de médecine occidentale, des soins de médecine orientale (également reconnue en Corée) et des soins dentaires. En 2002 est créée à Wonju, à l'initiative des mouvements coopératifs déjà existants, une cinquième coopérative médicale à laquelle adhèrent aujourd'hui environ 2500 familles. La coopérative médicale de Wonju emploie près de 80 personnes, la majeure partie d'entre elles dans le domaine des soins à domicile aux personnes âgées pris en charge dans le cadre de l'assurance grande dépendance (long-term care insurance), introduite en Corée en 2008.

Ces premières expériences de coopératives médicales amènent en 2003 la création de la Fédération nationale des coopératives médicales. Leur démarche met en exergue une vision alternative de l'exercice de la médecine, reposant sur la volonté de développer une médecine de proximité génératrice de lien social, d'une part en s'adressant à des populations en difficulté, d'autre part en prônant vis-à-vis de tous leurs usagers un modèle de médecin de famille plus disponible que ne le sont habituellement les médecins, dont on estime qu'ils réalisent en Corée autour de 70 consultations par jour (étude de 2010). Les coopératives médicales mettent également l'accent sur la nécessité de diminuer l'usage des antibiotiques (dont la Corée est l'un des plus gros consommateurs) et sur l'importance de la prévention et du dialogue entre médecin et patient. Les coopératives médicales offrent aux médecins qu'elles emploient des conditions de travail sensiblement différentes, qui se traduisent par une rémunération moins élevée que la moyenne observée dans les autres établissements (en tant que médecins salariés ou en tant que médecins indépendants) mais des horaires de travail beaucoup moins contraignants. Au-delà de l'arbitrage classique entre revenu et loisir, exercer dans une coopérative médicale implique également, de la part du médecin, de rompre avec la posture habituelle de la profession qui laisse peu de place au dialogue avec le patient. En tant que tel, il s'agit donc d'un modèle porteur d'une approche alternative de l'exercice de la médecine et de la délivrance des soins.

En matière de santé, la Corée du Sud a mis en place à la fin des années 1970 un système d'assurance maladie obligatoire, qui a été progressivement étendu à l'issue de réformes successives. Au fil de ces réformes, le choix a été fait de privilégier un taux de couverture élevé (97\% de la population est couverte) et un bas niveau de cotisation obligatoire (6\% environ des salaires, versés à parts égales par l'employeur et l'employé). Il en résulte mécaniquement un niveau modéré de prise en charge des soins 
et un reste à charge moyen assez élevé pour l'assuré (de l'ordre de $35 \%$ des dépenses de santé contre 7,5\% en France selon l'OCDE). Pour $45 \%$ (contre $28 \%$ en moyenne dans l'OCDE), les dépenses de santé relèvent d'un financement privé. L'offre de soins est libérale et quasi intégralement assurée par des établissements privés à but lucratif (cliniques et cabinets médicaux) ou non lucratif (essentiellement des fondations et, de manière beaucoup plus marginale, des coopératives médicales). Les éléments de comparaison statistique montrent que la Corée se distingue par une espérance de vie à la naissance supérieure à la moyenne de l'OCDE, mais des valeurs très supérieures en matière de reste à charge pour les malades (deuxième niveau plus élevé au sein des pays de l'OCDE), de proportion de prestations non couvertes par le champ de l'assurance maladie, de prescription de traitements antibiotiques, de nombre de consultations par praticiens ou de durée moyenne d'hospitalisation.

Le modèle que proposent les coopératives médicales s'inscrit dans une démarche qui vise à promouvoir une approche différente, privilégiant la recherche d'une réponse participative et responsable à des problématiques locales liées à la santé et à la pauvreté (Bidet, Eum, 2014). Ce modèle traduit le choix de communautés locales d'organiser elles-mêmes une réponse à une problématique de santé et de cohésion sociale sur un territoire en favorisant l'accès aux soins de catégories en difficulté, en mettant l'accent sur la prévention et en privilégiant la prescription de traitements moins coûteux pour le patient et pour le système d'assurance maladie. Les coopératives médicales conjuguent donc l'intérêt mutuel, en ayant pour finalité de proposer des soins de santé répondant aux besoins de leurs adhérents, et l'intérêt général, en poursuivant l'objectif de permettre l'accès aux soins à des catégories exclues du système de soins et de contribuer à réduire l'enveloppe globale des dépenses de santé, au moyen de la prévention et de traitements moins coûteux pour la collectivité. Si les modalités de mise en œuvre ne sont pas tout à fait les mêmes, ce sont des objectifs qui rejoignent ceux défendus en France par les mutuelles à travers la mise en place de centres de soins mutualistes ou, plus récemment, leur mobilisation pour les médicaments génériques. C’est en vertu de cette finalité sociale que les coopératives médicales ont pu être reconnues comme entreprise sociale, au titre de la loi de 2006 sur la promotion de l'entreprise sociale.

Dans le contexte coréen, le modèle de la coopérative médicale, même s’il reste relativement marginal, est intéressant à différents égards. Par rapport aux autres formes d'entreprises sociales reconnues par la loi de 2006, elles incarnent une forme d'organisation offrant une plus grande pérennité que nombre d'autres entreprises sociales agréées, dont la survie devient difficile dès lors que diminue ou disparaît le soutien public 
accordé pour une durée de trois ans. Elles se distinguent également des autres formes d'entreprises sociales par leur gouvernance participative. Les coopératives médicales ont donc suscité beaucoup d'intérêt comme modèle d'entreprise sociale fournissant des services sociaux professionnels, ayant une taille assez importante et appliquant une gouvernance démocratique et participative en tant que coopérative. Par rapport aux autres formes de coopératives, les coopératives médicales représentent la première tentative de coopérative à sociétariat multiple. Une de leurs particularités est en effet d'associer, dans leur fonctionnement et dans leurs organes de contrôle, différentes catégories de sociétaires telles que des professions médicales, des représentants de la société civile et des usagers, y compris quelques fois, mais plus rarement, des personnes issues de catégories en difficulté (personnes handicapées, personnes âgées, femmes, paysans, citoyens pauvres, etc.).

Enfin, dans le cadre plus général du système de soins coréen, les coopératives médicales représentent l'une des rares tentatives pour mettre en place une gouvernance participative, dans un système de santé où les professions médicales contrôlent une offre de soins très marchandisée. Une telle gouvernance participative apparaît comme l'une des voies à explorer pour enrayer la tendance persistante à l'augmentation des dépenses de santé sous le double effet du vieillissement rapide de la population et du comportement opportuniste des professions médicales pour générer des profits plus élevés.

Les quatre principales coopératives médicales (Ansan, Anseong, Incheon et Daejeon) assurent les deux tiers environ de l'activité de l'ensemble des coopératives affiliées à la fédération nationale et rassemblent environ $55 \%$ du nombre total des associés (17000 sur un total de 32000 en 2013). Les plus importantes (Ansan et Anseong) touchent 5000 à 6000 familles et emploient jusqu'à une quinzaine de médecins et une centaine de personnes (infirmiers, kinés, travailleurs sociaux, personnel administratif) chacune; les plus petites regroupent quelques centaines de familles autour d'un ou de quelques médecins. A Anseong, on estime que $10 \%$ environ de la population totale est affiliée à la coopérative médicale qui propose, au-delà des soins médicaux, toutes sortes d'activités telles que des cours de langue ou des activités sportives et culturelles. Certains coopératives médicales ne proposent que des soins de médecine occidentale, mais la plupart ont fait le choix de proposer différents types de soins, notamment des soins à domicile entrant dans le cadre de l'assurance grande dépendance. Jusqu'en 2010, les coopératives médicales étaient confrontées à un dilemme fondamental: la loi de santé interdit de refuser des patients dont la situation sanitaire exige une prise en charge, tandis que la loi coopérative interdisait de délivrer des services à des personnes 
non associées (members only rule). Cela limitait leur développement et rendait d'autant plus difficile leur mission sociale en direction des populations en difficulté. La modification de la loi sur les coopératives de consommation introduite en 2010 (et inspirée par l'exemple japonais) a harmonisé cette situation en permettant que des utilisateurs non associés puissent représenter jusqu’à $50 \%$ des bénéficiaires.

Une autre difficulté majeure à laquelle devaient faire face les coopératives médicales était l'utilisation massive du nom par des organisations ne partageant ni les mêmes valeurs ni les mêmes objectifs. En tant que forme particulière de coopérative de consommateurs, la coopérative médicale ne bénéficiait en effet pas de mesures protectrices particulières autres que les dispositions générales prévues par la loi sur les coopératives de consommateurs. Selon cette loi, quiconque réunissant le capital initial nécessaire (environ 25000 euros) et rassemblant au moins 300 utilisateurs est autorisé à créer une coopérative de consommateurs dont l'objet peut être la distribution de soins médicaux. Un grand nombre d'investisseurs, voyant dans les soins de santé un secteur particulièrement rentable, ont donc utilisé ce cadre juridique pour ouvrir des centres de soins et contourner la loi relative à la santé qui limite la possibilité d'ouvrir une clinique ou un cabinet médical à un professionnel de santé. Sur les quelque 300 coopératives médicales légalement inscrites en tant que telles en 2011, une vingtaine seulement étaient affiliées à la Fédération des coopératives médicales et considérées à ce titre comme de véritables coopératives médicales, les autres étant assimilées à de "fausses » coopératives médicales. Il a fallu attendre la loi coopérative générale de 2012 pour apporter une réponse à cette utilisation détournée du modèle coopératif.

\section{La reconnaissance du modèle coopératif}

La loi coopérative générale de 2012 permet de constituer une coopérative dans n'importe quel secteur d'activité, excepté la finance et l'assurance (Statutes of the Republic of Korea, 2012). L'article 3 de la loi précise bien qu'il s'agit de promouvoir des coopératives indépendantes et autonomes, en accord avec les principes coopératifs de l'ACI. Son adoption traduit tout d'abord le fait que le modèle coopératif a été identifié par les autorités publiques sud-coréennes comme un modèle potentiellement créateur d'emplois en référence à ce que l'on appelle « l'indicateur d'emplois induits » (Employment Inducement Coefficient), qui montre un ratio emplois-capital investi nettement plus élevé dans les coopératives et les entreprises sociales que dans les autres formes d'entreprises. En ce sens, la loi coopérative de 2012 s'inscrit dans la continuité des dispositifs précédents, en premier lieu motivés par la lutte contre le chômage et la création d'activités. 
Au-delà de cette continuité, la loi de 2012 a considérablement modifié la situation et les formes du mouvement coopératif coréen. Elle consacre tout d'abord l'obligation pour les pouvoirs publics d'avoir une politique publique vis-à-vis des coopératives (art. 3: Master Plan for Policies on Cooperatives) révisée tous les trois ans sous l'égide d'un conseil politique de la coopération (art. 4: Cooperatives Policy Council) dans lequel figurent des représentants des principaux organes publics concernés et des personnes qualifiées désignés par le ministre de la Stratégie et des Finances. Dans un pays marqué par un strict cloisonnement entre les mouvements coopératifs existants, cela constitue un premier pas vers une forme d'intercoopération dont il faudra voir si elle inclura également les mouvements coopératifs existant déjà sous d'autres cadres juridiques. La loi de 2012 vient parachever un processus de transformation amorcé avec le développement des coopératives de consommation en apportant une reconnaissance au modèle de l'entreprise coopérative et en modifiant son image, qui était celle d'une organisation quasi publique. Cette loi, qui ne vient pas se substituer mais compléter les lois existantes régissant les coopératives du secteur primaire, de la banque et de consommateurs, distingue deux types complémentaires de coopératives: la coopérative sociale et la coopérative « générique ».

Pour une large part (articles 11 à 23), la loi de 2012 est spécifiquement consacrée aux coopératives sociales. L’article 12 précise qu'une telle coopérative doit réunir au moins cinq membres relevant de différentes catégories de sociétaires, telles que producteurs, consommateurs, employés, bénévoles ou bénéficiaires. Il s'agit donc d'une coopérative à sociétariat multiple, à l'instar de la Scic en France, mais n'offrant pas la possibilité d'associer une collectivité publique en tant que catégorie spécifique. L’article 14 (Criteria and Method for Determination of Main Business) identifie deux types d'activités qui peuvent justifier la création d'une coopérative sociale: d'une part des activités répondant à des besoins propres à un territoire (revitalisation d'un territoire, maintien d'une activité économique, amélioration de l'environnement local, réponse à des besoins rencontrés localement, etc.), d'autre part des activités destinées à fournir des emplois ou des services sociaux (dans les domaines de l'éducation, de la santé, de l'environnement, de la culture, etc.) à des catégories en difficulté. Il s'agit donc d'une forme de coopérative dont l'activité présente une utilité sociale, telle que définie en France par la loi ESS de 2014. La coopérative sociale est par ailleurs une coopérative à but non lucratif, qui ne peut distribuer ses excédents à ses associés et doit en consacrer au moins $30 \%$ à des réserves collectives. Contrairement aux coopératives " génériques ", qui sont déclarées et enregistrées auprès de collectivités locales, l'obtention du statut de coopérative sociale est soumise à une autorisation 
ministérielle et procure des avantages fiscaux spécifiques, liés à la non-lucrativité. Début 2016, on dénombre plus de 400 coopératives enregistrées comme coopératives sociales. Les deux tiers d'entre elles ont été habilitées par le ministère de la Santé (25\%), le ministère de l'Education ( $20 \%$ ) ou le ministère du Travail (20\%). L'article 12 de la loi précise qu'à condition de respecter certaines règles spécifiques, une coopérative sociale peut s'engager dans une activité de soins médicaux: elle doit alors réunir au moins 500 membres qui doivent s'acquitter chacun d'une contribution d'au moins $50000 \mathrm{KRW}$ (40 euros) pour constituer un capital d'au moins 100 millions de KRW (80000 euros), sans qu'aucun associé n'apporte à lui seul plus de $10 \%$ de l'ensemble des contributions. Les mesures introduites par la loi de 2012 ont amené les coopératives médicales à se transformer en coopératives sociales, alors qu'elles représentaient auparavant une forme particulière de coopérative de consommateurs, soumise depuis 2010 à des restrictions concernant la répartition de ses excédents. Cela a eu pour effet de dissiper la confusion qui existait entre "fausses » coopératives médicales, qui continuent à exercer en utilisant le statut juridique de coopérative de consommateurs, et "vraies » coopératives médicales, qui opèrent désormais avec le statut de coopérative sociale engagée dans une activité de soins médicaux.

A travers ce qu'elle considère comme des coopératives "génériques ", la loi de 2012 offre notamment une première reconnaissance légale au modèle de la coopérative de travailleurs, jusqu'alors ignoré dans le droit coréen (Kim, 2013). Dans l'esprit de la loi, cela renvoie aussi bien à des travailleurs salariés que non salariés (notamment des travailleurs indépendants ou entrepreneurs individuels). L’intérêt en Corée pour le modèle de la coopérative de travailleurs ne date pas de l'adoption de la loi de 2012, puisqu'il est apparu dès la fin des années 1980, suscitant notamment la création de démarches collectives pour la récupération d'entreprises en difficulté par leurs salariés, pour la création d'activités par des populations - notamment féminines - longtemps éloignées du marché du travail ou pour la mise en place de services sociaux et culturels dans des quartiers défavorisés. Il s’agissait donc de démarches fortement motivées par des problématiques sociales (maintien ou accès à l'emploi, accès de populations pauvres à certains services). Cependant, dans un contexte socio-politique encore très hostile à toutes formes d'organisations des travailleurs, c'est un modèle qui est resté très marginal, le plus souvent sous la forme de collectifs de travail issus du mouvement social et fréquemment organisés pour répondre à des problématiques très territorialisées, avec comme modèle de référence celui de Mondragon, au Pays basque espagnol. Beaucoup de ces initiatives ayant comme principal moteur la volonté d'apporter un emploi à des chômeurs en développant 
des activités commerciales ou industrielles, elles ont, dans un premier temps, inspiré certains dispositifs publics de traitement du chômage, notamment le programme de self-sufficiency mis en place au début des années 2000 (Eum, Bidet, 2014).

Si elle n'apporte pas à la coopérative de travailleurs une pleine reconnaissance légale à travers un cadre juridique spécifique, comme peut l'être en France la loi de 1978 complétée par la loi sur l'ESS de 2014, la loi coopérative de 2012 offre à la coopérative de travailleurs une première reconnaissance significative. Elle contribue par ailleurs à la déconnecter d'une vision uniquement sociale pour en faire un modèle d'entreprise adapté à toutes sortes d'activités. En mai 2015, on estimait que cette loi avait suscité la création d'environ 300 coopératives pouvant être considérées comme coopératives de travailleurs - il est difficile de donner un chiffre précis, dans la mesure où n'apparaît pas de catégorie spécifique dans les statistiques du ministère de la Stratégie et des Finances.

La plus développée, Happy Bridge, développe une activité de restauration à travers un réseau national de franchisés qui rassemble plus de 500 restaurants représentés dans sept chaînes, dont la plus importante est Noodle Tree (littéralement: « L'arbre à nouilles »). La coopérative, qui est née de la transformation d'une société de capitaux classique en coopérative à la faveur de la loi de 2012, emploie 120 personnes environ, dont les deux tiers sont associées. L'ouverture du capital à des non-associés est limitée à $30 \%$, l'apport minimal en capital est de 50 millions de won (40 000 euros environ), ce qui est censé représenter l'équivalent d'une année de salaire. Les excédents sont répartis de sorte que 30 \% soient affectés aux réserves impartageables, $35 \%$ provisionnés pour les investissements, le reste réparti entre associés et salariés associés. En marge de son activité de restauration, Happy Bridge a également développé un institut du management coopératif, en partenariat avec Mondragon, et joue un rôle clé dans l'institutionnalisation du mouvement des coopératives de travailleurs, puisque le président de Happy Bridge est le premier président de la Fédération nationale des coopératives de travailleurs, fondée en 2014.

D’après les statistiques du ministère de la Stratégie et des Finances, près de 9000 coopératives (dont $40 \%$ environ à Séoul et dans la région limitrophe du Gyeonggi) étaient enregistrées début 2016 en référence à la loi 2012, ce qui représente une moyenne de 250 coopératives enregistrées chaque mois. Parmi elles, 25 à $30 \%$ avaient une activité dans le secteur de la distribution et du commerce, 10 à $15 \%$ dans l'agriculture, la pêche et les forêts, 10 à $15 \%$ dans l'éducation et les services, 8 à $10 \%$ dans la production manufacturière, 5 à $10 \%$ dans le sport et les loisirs. Il n'est pas faux de dire que la loi de 2012 a généré un véritable « choc coopératif », mais cette appréciation mérite 
d'être nuancée : d'une part, parce qu'on estime que seulement la moitié des coopératives enregistrées sont effectivement actives; d'autre part, parce qu'un nombre important de ces coopératives (les deux tiers environ) sont des coopératives de PME, d'entrepreneurs individuels ou de travailleurs indépendants, sous une forme qui ne diffère pas toujours de celle d'une association professionnelle. Il convient de noter également, comme l'illustre l'exemple de Happy Bridge, qu'il ne s'agit pas uniquement de créations ex nihilo mais souvent de transformations en coopératives (ce qui n'enlève rien à l'attrait du modèle coopératif ainsi défini par la loi).

Sur une période deux fois moins longue, la loi 2012 a donné lieu à six fois plus d'enregistrements que la loi de 2006 pour la promotion de l'entreprise sociale (1500 entreprises agréées). Bien entendu, les deux lois n'offrent pas le même potentiel, puisque l'une ne définit pas un statut d'entreprise spécifique mais apporte une reconnaissance (et une labélisation) à des entreprises existantes engagées dans une activité à finalité sociale, tandis que l'autre propose un statut d'entreprise spécifique susceptible d'être utilisé pour mener toute forme d'activité économique (à l'exception de la banque et de l'assurance). Dans un pays où ont longtemps prévalu des valeurs parfois contradictoires avec celles qui fondent le modèle coopératif (Bidet, 2009), il sera intéressant de voir, dans les années qui viennent, les modalités de fonctionnement concrètes que va prendre la gouvernance participative, en particulier dans les coopératives de PME, d'entrepreneurs individuels ou de travailleurs indépendants, qui représentent plus des deux tiers des coopératives créées.

\section{Conclusion}

L'expérience coréenne nous montre comment les évolutions successives du mouvement coopératif depuis deux décennies ont modifié, enrichi et réhabilité l'image de la coopérative. D'abord essentiellement placée sous la tutelle du gouvernement, comme la plupart des organisations durant la période de dictature (1948-1987), la coopérative a longtemps été assimilée à une forme d'agence publique. L'émergence, le développement puis la reconnaissance légale d'initiatives issues du mouvement social et échappant au contrôle gouvernemental ont remis en question cette perception, d'abord sous l'influence des coopératives de consommation qui s'organisent dans les années 1980 et sont reconnues légalement en 1999, puis à travers les coopératives médicales qui émergent dans les années 1990 et sont mises en avant comme formes d'entreprises sociales exemplaires par la loi de 2006, enfin grâce aux nouvelles formes de coopératives (en particulier les coopératives sociales et les coopératives 
de travailleurs) apparues avec la loi de 2012. Celle-ci offre la possibilité de créer et de gérer une coopérative avec plus de flexibilité et d'autonomie.

Bien que leur développement soit resté assez modeste (une vingtaine de structures couvrant de 300 à 6000 familles selon les cas), il faut souligner le rôle particulier joué par les coopératives médicales adoubées par la loi de 2006 pour la promotion de l'entreprise sociale, et juridiquement enregistrées en référence à la loi de 1999 sur les coopératives de consommation. Ce double référencement à des sphères qui restaient jusqu'alors très cloisonnées a contribué à unifier la nébuleuse d'acteurs rassemblés depuis le milieu des années 2000 autour du concept d'entreprise sociale et le mouvement des coopératives de consommateurs, qui s'est institutionnalisé à la fin des années 1990. Cette réunion est l'un des éléments qui a permis à l'émergence du concept d'économie sociale à partir de la fin des années 2000, alors que celui-ci était encore très peu considéré en Corée une dizaine d'années auparavant (Bidet, 2000). On a alors vu apparaître des réflexions de chercheurs coréens très liés aux acteurs du terrain sur l'application en Corée du concept européen d'économie sociale et sur la définition de l'économie sociale dans le contexte coréen. Cette réflexion a associé des représentants des mouvements sociaux engagés dans la lutte contre le chômage et la précarité, mais également des acteurs du mouvement des coopératives de consommateurs et d'une partie des credit-unions.

Dans les instituts nationaux de recherche, on a développé des études plus rigoureuses sur les perspectives de créations d'emploi dans le tiers secteur en Corée ou sur la promotion du tiers secteur en Corée. Cette réflexion a ensuite touché les décideurs locaux, jusqu'à conduire la plupart des collectivités territoriales à mettre en place des " centres d'économie sociale » (l'un des premiers, Chungnam Social Economy Center, est celui créé en 2011 à Gongju à 80 kilomètres au sud de Séoul; le plus important, Seoul Social Economy Center, est celui ouvert à Séoul deux ans plus tard) et à faire voter des décrets locaux pour la promotion de l'économie sociale. On évoque même depuis deux ans la possibilité que soit adoptée une loi nationale pour l'économie sociale, qui ferait de la Corée l'un des rares pays à se doter d'une telle loi. Quoi qu'il advienne de ce projet de loi et quels que soient ses contours, la Corée est un pays où l'on assiste, depuis une quinzaine d'années, à une forte reconnaissance - notamment publique et académique - des concepts d'entreprise sociale et d'économie sociale, qui se sont nourris l'un et l'autre jusqu'à pouvoir être compris comme différentes expressions d'un même objet (Eum, Bidet, 2014). Les évolutions récentes montrent que le modèle coopératif tend à devenir un des principaux modèles de référence pour le développement 
d'activités économiques alternatives, privilégiant l'homme plutôt que le capital, l'esprit de coopération et de réciprocité, plutôt que l'esprit individualiste.

Au niveau européen et plus encore au niveau mondial, on sait que l'expérience française est emblématique d'un intérêt précoce pour l'économie sociale qui, sans remonter jusqu'aux racines historiques du XIX ${ }^{\mathrm{e}}$ siècle, émerge en France au milieu des années 1970 et rencontre une première reconnaissance institutionnelle dès le début des années 1980. En même temps qu'un approfondissement, qui se traduit par la mise en place d'un environnement institutionnel complexe associant tout un ensemble d'institutions et de dispositifs nationaux et régionaux, ce mouvement s'est accompagné en France d'un élargissement du périmètre de l'économie sociale, qui est progressivement devenue économie sociale et solidaire et vise également à intégrer, depuis la loi de 2014, certaines entreprises engagées dans des activités d'utilité sociale. En comparaison, l'expérience coréenne témoigne d'un intérêt plus tardif et surtout d'un cheminement inverse, qui part d'une volonté de promouvoir le modèle de l'entreprise sociale (ou entreprise à finalité sociale) pour aboutir à l'émergence du concept d'économie sociale dont la reconnaissance institutionnelle pourrait prochainement se traduire, comme en France, par l'adoption d'une loi spécifique sur l'économie sociale, qui intégrera l'entreprise sociale et d'autres formes d'entreprises à gouvernance ou à finalité spécifique. La mise en perspective de ces deux cheminements nous montre combien la question de la relation entre les concepts d'entreprise sociale et d'économie sociale est complexe, mouvante et ancrée dans chaque contexte particulier. - 


\section{BIBLIOGRAPHIE}

Bidet E., 2000, "Economie sociale et NPI : le cas de la Corée du Sud ", Annales de l'économie publique et coopérative, vol. $71, \mathrm{n}^{\circ} 3$.

Bidet E., 2009, « La difficile émergence de l'économie sociale en Corée du Sud ", Recma, $n^{\circ} 310$, p. 65-78.

Bidet E. et Eum H., 2014, " Nouvelles formes de protection sociale : entreprises sociales et coopératives médicales en Corée du Sud ", Revue française des affaires sociales, $\mathrm{n}^{\circ} 3$.

Eum H. et Bidet E., 2014, " Dynamiques de l'économie sociale en Corée du Sud ", Recma, $\mathrm{n}^{\circ} 332$
Eum H., 2008, "Social Economy and Social Enterprise in South Korea : Comparison with European Experiences and their lessons ", Work Together Foundation (en coréen).

Kim H., 2013, " Flux on Korean Consumer Cooperative Activities: The experience of consumer cooperative movement in Korea: Its break and rebirth ", APAY Regional Workshop on Social Economy.

Kim S., 2013, " The Cooperative Movement in Korea ", Journal of Global Management, vol. $25, \mathrm{n}^{\circ} 2$. 